\title{
Elastic-plastic Finite Element Simulation of Shear Spinning of Cone Part
}

\author{
Qin Sheng-xue ${ }^{1}$, Chen Bao-cheng ${ }^{1}$, Liu Jie ${ }^{1}$, Chen Chao ${ }^{2}$ \\ ${ }^{1}$ Shandong University of Science and Technology, College of Mechanical and Electrical Engineering, Qingdao, 266590; \\ ${ }^{2}$ Taikai Electric Group Co., Ltd., TaiAn, 271000 \\ qinshengxue01@163.com
}

\begin{abstract}
In order to make spinning computer simulation correspond with the actual production, the elastic-plastic finite element model of spinning was established in this paper. By using DEFORM, the elastic-plastic and rigid-plastic finite element models of cone spinning were developed, and the results of stress and strain distribution of the two models were compared. Furthermore, the relationship of the rotating rollers force with the roller nose radius was given. Research shows that the two models have the same largest equivalent stress, but some differences in stress and strain distribution; the strain and its distribution area are smaller than those with the model developed by elastic-plastic FEM method; the spinning roller force will increase with the roller nose radius increasing.
\end{abstract}

Index Terms - Spinning; Finite element; DEFROM; Elasticplastic model

\section{Introduction}

Metal spinning, also known as spin forming or spinning or metal turning most commonly, is a metal working process by which a disc or tube of metal is rotated at high speed and formed into an axially symmetric part. As in [1,2], spin forming process can be used for metal forming with large deformation, and has some advantages, such as smaller work force needed, saving materials and the products have higher strength. This process is widely used in aerospace industry, missile production, chemical industry and automobile industry, et al.

As in Ref. [3], the rule of metallic flow in spin forming could be investigated by FEM simulation; stress and strain change history of workpieces also could be obtained. Those data are contribute to study on the affection of process parameters on the metal forming performance and quality. Reference [4,5] was that, in recent years, many researchers have studied spin forming process by using fem simulation, and have made appreciable advances in spin forming mechanism analysis, stress state and process parameters optimization. As in Ref. [6,7], in spin forming process, the elastic deformation is much less than the plastic deformation, so the elastic deformation is often ignored to simplify the analysis model. While, the simplify model resulted in the affection of elastic deformation on the forming process could not be considered. In spin forming, the moving passes of the spinning roller is a very important factor to affect forming quality and the service life of the roller, while the elastic

* This work is partially supported by A Project of Shandong Province Higher Educational Science and Technology Program \#J12LB03 to Qin shengxu deformation of the workpiece will affect the design of the moving passes of the spinning roller. For this reason, the moving passes of the spinning roller should be compensated by considering the elastic deformation of the workpiece, so the forming process could be controlled more accurately and longer service life of the roller could be obtained.

In this paper, the spin forming analysis model is established by using elastic-plastic FEM theory and a cone forming process was simulated with elastic-plastic and rigidplastic material model respectively. The stress and strain distributions were investigated in two models, and the affection of the elastic deformation on forming process was discussed. The force of the spinning roller was further studied based on the two models. The simulation results are valuable to instruct the spin forming process and spinning roller design.

\section{Elastic-plastic finite element method theory}

Ref. [8] shows, in 1965, the elastic-plastic finite element method was proposed and used in structure and stress analysis by Marca. After that, based on small deformation assumption, more and more researchers used the elastic-plastic finite element method to solve the problem of metal forming. Metal plastic forming is an elastic-plastic problem with large displacement deformation and finite strain, the assumption of small deformation will increase the error as the deformation increase. Therefore, the theory based on the finite element deformation theory that could be used to describe the precise large displacement, large rotation, large strain got more and more attention.

According to the stress-strain relationship of elasticplastic mechanics, with a small force, the material is in elastic state when the equivalent stress is smaller than the elastic limit. When the equivalent stress of some spots reaches to the elastic limit as the force increases, these spots in the material enter the plastic state. This theory could be described as equation (1).

$$
d \varepsilon=d \varepsilon^{e}+d \varepsilon^{p}
$$

there, $e$ represents elastic and $p$ represents plastic. In elastic stage, the stress strain relationship follows Hooke's law, and the strain depends on the final stress state. In elasticplastic stage, when the equivalent stress reaches the yield strength, the stress-strain relationship is determined by the elastic-plastic matrix $D_{e p}$ : 


$$
\Delta \sigma=D_{e p} \Delta \varepsilon
$$

The Lagrangian description method for finite Elasticplastic deformation is also known as particle coordinate system description. Using the initial form pictured as the reference, the Virtual power equation is

$$
\int_{0} S_{i j} \delta \dot{E}_{i j} d V_{0}=\int_{S_{0 \sigma}} p_{i}^{0} \delta v_{i} d S_{0}+\int_{\nu_{0}} F_{i}^{0} \delta v_{i} d V_{0}
$$

there, $V_{0}$ is the volume in the initial state; $S_{0}$ is the surface area in the initial state; $p_{i}^{0}$ is component of the stress vector on the surface $S_{0 \sigma}$ in the initial state; $F_{i}^{0}$ is the force in the initial state; $E_{i j}$ is state of strain of the point in the object; $\delta v_{i}$ is Virtual velocity of the point in the object.

According to the formula (3), when plastic deformation occurs, the stress is not only related to strain but aslo material microstructure and deformation history. Therefore, the relationship between the stress and strain is no longer a linear and single value.

\section{The development of the finite element model}

Spin forming is a typical metal forming technology with large deformation and strain. In this paper, the simulation model was developed by using DEFORM based on the plastic-elastic theory with large deformation. The cone spinning process with single roller was simulated and discussed. The material of workpiece is AISI 1010, and the constitutive equation is described as equation (4).

$$
\bar{\sigma}=c \varepsilon \dot{-} \dot{\bar{\varepsilon}}+y
$$

there, $c$ is strength coefficient; $n$ is hardening exponent, $0<n<1$.

The curve of flow stress in DEFROM is shown in Fig.1.

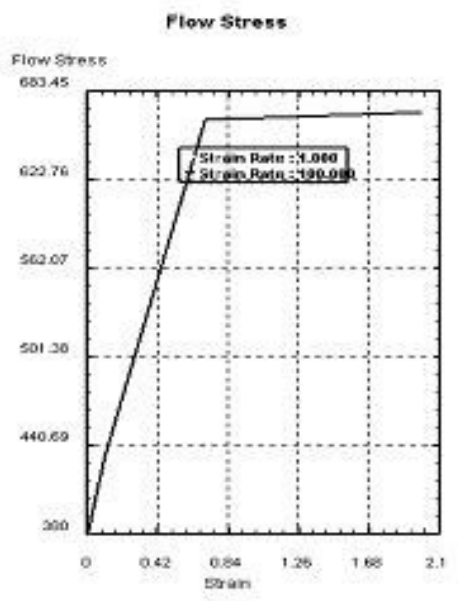

Fig.1 Flow stress

The discrete model of the workpiece include 25000 elastic-plastic elements. Using relative motion way to describe the relationship of the parts. As shown in Fig.2, the sheet blank is mounted on the rotating mandrel, and the spinning roller rotates around the rotating shaft of mandrel. At the same time, the spinning roller is feeding in one direction. The simulation process was divided into 5000 steps, each step time is $0.002 \mathrm{~s}$. The roller diameter is $76 \mathrm{~mm}$, fillet radius is $38 \mathrm{~mm}$, roller nose radius is $19 \mathrm{~mm}$, half cone angle is $45^{\circ}$, the workpiece diameter is $152 \mathrm{~mm}$, and thickness is $3 \mathrm{~mm}$.

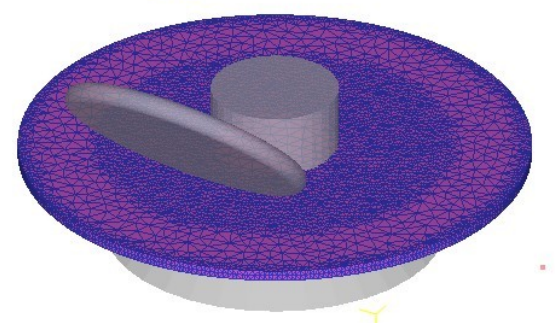

Fig.2 finite element model

\section{Results and discussion}

A. Finite element analysis of elastic-plastic model

Fig. 3 and Fig. 4 show the equivalent stress distribution of elastic-plastic model at the 1000th step and 5000th step. As shown in the figures, with the increase of deformation, stress distribution is in outward diffusion; In the place of roller contact with the billet.

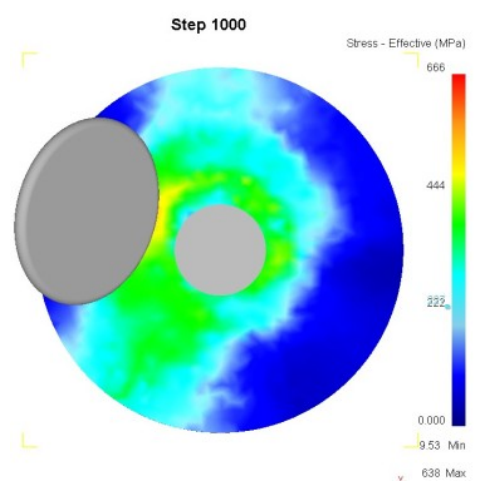

Fig. 3 equivalent stress distribution at 1000th step

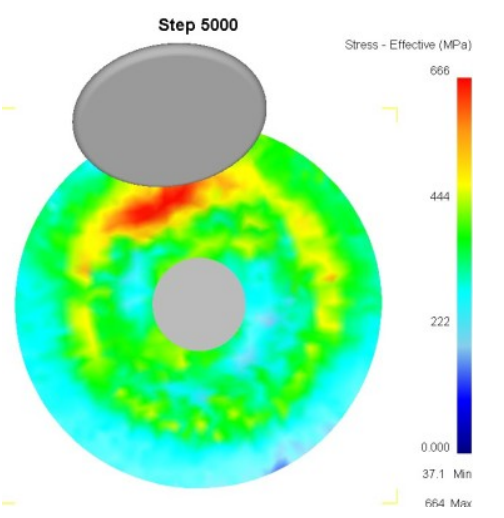

Fig.4 equivalent stress distribution 5000th step 
Fig.5-Fig.8 are the equivalent stress and equivalent strain distribution at the 1500th step of rigid-plastic and elasticplastic model respectively. Fig.5 and Fig.6 show that the same maximum equivalent stress $(660 \mathrm{Mpa})$ of the two models both appear in the roller working region. This is because the two models have same yield strength. But the equivalent stress distribution is different in the two models when the model is rigid-plastic, the axial symmetry of the distribution of equivalent stress is not very obvious. Compared with the elastic-plastic model analysis, the difference of equivalent stress between working range and its symmetric region is large, and the stress is all in the roller working region. In the elastic-plastic model analysis, the larger equivalent stress appears in the roller working region. Compared with the rigidplastic model, its equivalent stress value on the axial symmetry area of the roller working-region is larger, that is because the equivalent stress in this area includes the elastic stress and plastic stress.

As shown in Fig.7 and Fig.8, the value and distribute area of equivalent strain of rigid-plastic model is larger than elastic-plastic model, that is because, in the rigid-plastic model, the workpiece are in two states, including the plastic deformation and no deformation, and the equivalent strain is the plastic strain. While in the elastic-plastic model the workpiece has three states, including no deformation, elastic and plastic deformation, so the strain value is the sum of elastic and plastic strain. Similarly, in the unloading area, the elastic part of the equivalent strain will be unloading in the elastic-plastic model analysis accordingly, the equivalent strain will decrease. It can be seen from comparing the analysis results of the two models that with the roller feeding, the difference of equivalent strain gradually increase from 0.347 in 1000th step to 0.6 in 1500th step, which verifies that when we use rigid-plastic model for analysis, the deformation error of workpiece will increase with the roller feeding on. In actual production, the moving passes of the roller will be changed due to the elastic recovery in the workpiece, so a positive displacement correction should be considered based on the strain difference between the rigid-plastic and elasticplastic analysis models for decreasing the affection of the recovery on the forming accuracy.

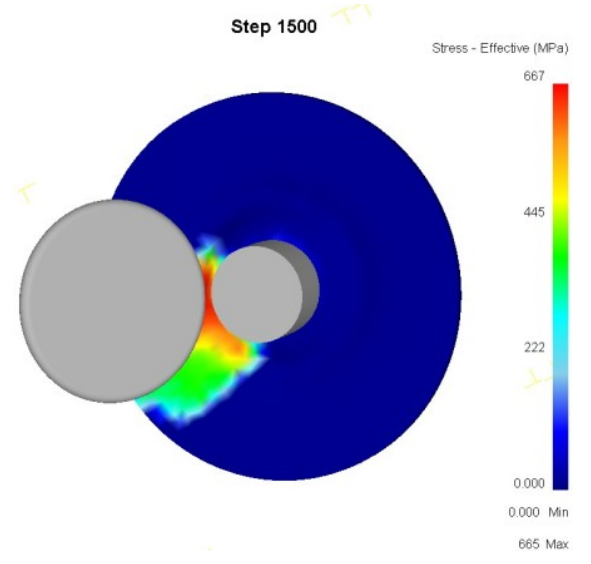

Fig.5 equivalent stress distribution at 1000th step of rigid-plastic model

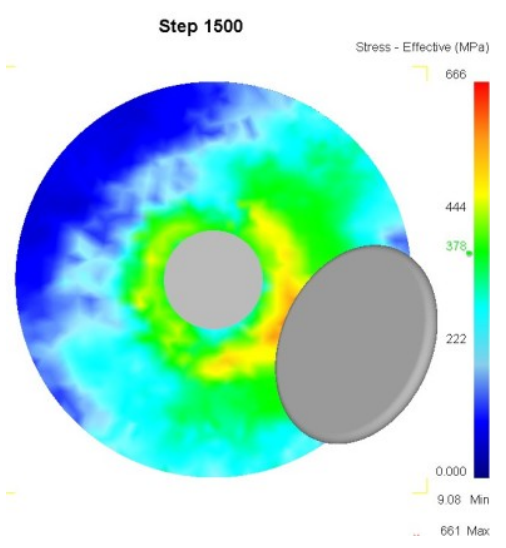

Fig.6 equivalent stress distribution at 1000th step of elastic-plastic model

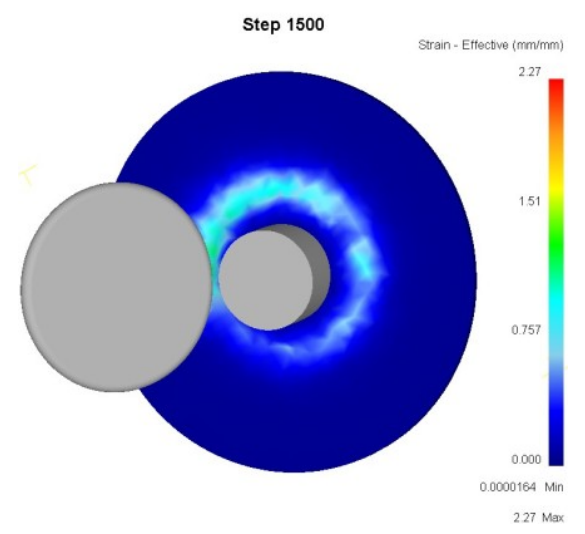

Fig.7 equivalent strain distribution at 1000th step of rigid-plastic model

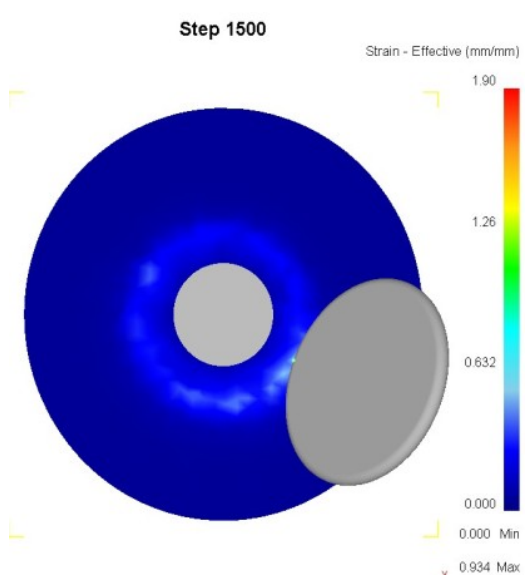

Fig.8 equivalent strain distribution at 1000th step of rigid-plastic model

\section{B. The analysis of the spinning roller force}

As in Ref. [9], spinning roller is a critical component for spin forming, and it is often damaged due to great extrusion stress and shear stress. The spinning roller belongs to wearing part in spin forming, so it is very important to study the stress state of the spinning roller to increase its work life. Fig.9 shows force of the roller. As seen in Fig.9, the roller's force will increase gradually with the movement of the roller. 


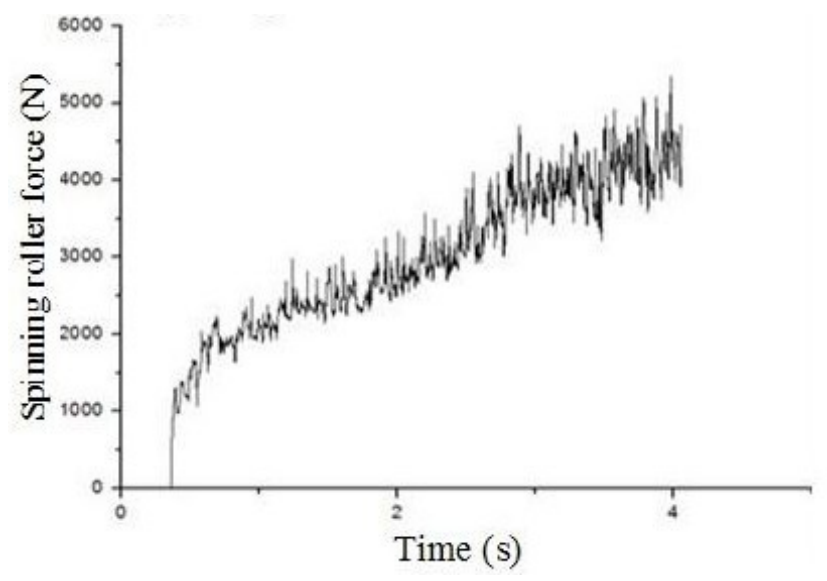

Fig.9 Spinning roller force $(\mathrm{N})$

In actual production, too big roller force can lead to inner stress exceeds the yield strength and result in the damage of the roller. According to the static analysis method of deformation model, the spinning force could be calculated by equation (5) by Ref. [10]

$$
F=(1 / 4) \sigma t_{0}\left[\left(r / r_{0}\right)-k\right]^{-1} \times\left[1+(1-2 k)^{2}\right](1+1 / k)^{1 / 2}
$$

There, $k=r_{1} / r_{0}$ is roller nose radius; $r_{0}$ is billet radius; $r$ is the radius of the area that equivalent stress acted on; $\sigma$ is yield limit; $t_{0}$ is the billet thickness, $r_{0}>r>r_{1}$

From equation (5), it can be found that the roller force is relative to the roller nose radius. In order to find out the affection of the radius on the roller force, several elasticplastic FEM models with different roller nose radius were developed by using DEFORM. Fig.10 shows the spinning roller cures when using three different roller nose radius. The roller nose radius larger is the greater, the force of the roller will be. The analysis results show that when the roller nose radius increase $5 \mathrm{~mm}$, the roller force will increase about $750 \mathrm{~N}$. So in actual production, we should select the appropriate roller nose radius for developing the roller life.

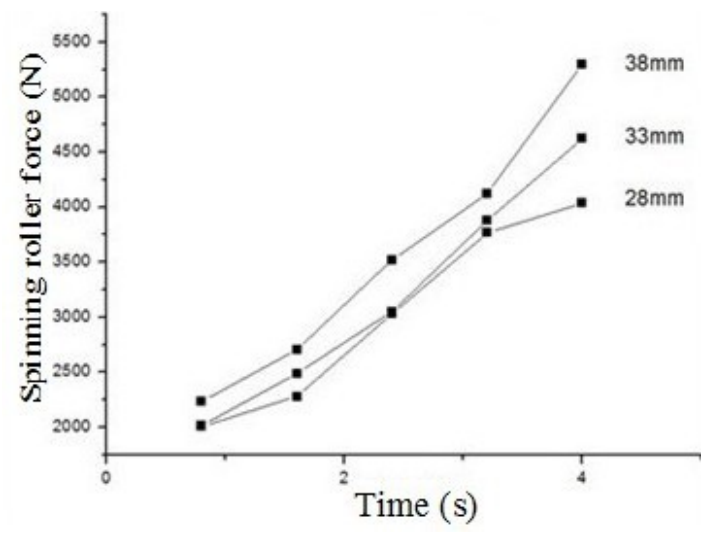

Fig.10 spinning roller cures when using three different roller nose radius

\section{Conclusions}

By using DEFORM, the cone part spinning process was simulated based on elastic-plastic FEM theory and the differences of the results between rigid-plastic and elasticplastic models were discussed. The distribution of stress and strain was analyzed. Through analyzing the spinning roller's force, the relationship between the force and the roller nose radius was obtained. The following conclusions have been drawn.

(1) The elastic-plastic model and the rigid-plastic model have the same maximum stress but different stress distribution. Compared with the rigid-plastic model, the stress distribution shows more axial symmetrical in elastic-plastic model, and the axial symmetric region of the spinning roller work area has larger stress.

(2) Relative to the rigid-plastic model, the elastic-plastic model has smaller equivalent strain value and less distribution area. With the roller feeding, the difference of equivalent strain of the two models becomes larger. As the deformation error of the workpiece will increase with the roller feeding gradually by using rigid-plastic model, a positive displacement correction should be considered based on the strain difference between the rigid-plastic and elastic-plastic analysis models.

(3) With the movement of the roller, the roller force increased gradually. With the roller nose radius increasing, the stress of the roller will increase.

\section{Acknowledgment}

This work was financially supported by A Project of Shandong Province Higher Educational Science and Technology Program( J12LB03).

\section{References}

[1] Wong C C, Dean T A, Lin J. "A review of spinning, shear forming and flow forming processes". International Journal of Machine Tools \& Manufacture, vol. 43, pp. 1419-1435, 2003

[2] Zhan Mei, Ma Ming juan, Yang He, et al. "Influence ofroller parameters on power spinning of thin-walled shell with special shape". Forging \& Stamping Technology, vol.5, pp. 144-147, 2006,

[3] Zhan Mei, Ma Shang Guan. "The Treatment of Relative Motion between Mandrel and Roller in Numerical Simulation for Tube Spinning". Journal of netshape forming engineering, vol.3, no, 6, pp. 107-111, 2011

[4] Liu Jian Hua, Yang He, Li Yu Qiang. "State of the art and trend of metal spinning technique". Heavy machinery, vol.3, pp. 1-4, 12, 2002

[5] Zhan M, Yang H, Zhang J H, et al. "3D FEM analysis of influence of roller feed rate on forming force and quality of cone spinning". Journal of Materials Processing Technology, pp. 187-188: 486-491, 2007

[6] Sun Li Li. "Numerical simulation on automobile hub spinning forming Process ". He Fei: HeFei University of Technology, pp. 24-35, 2008

[7] Li Lu Lu, Du Mao Hua, Bai Yu Feng. "Numerical Simulation Analysis of Backward Spinning Process for Metallic Cylinders". Casting Forging Welding, vol. 39, no. 9, pp. 115-119, 2010

[8] Ma Yu Ting. "Research on hot extrusion techniques and equipment for axle-shaft sleeve of engineering Machinery". Ji Nan: Shan Dong University, pp. 6-7, 2011

[9] Qin Sheng Xue, Ren Li Xin. Hao Ji Dong, et al. "Key technical problems of roller design and manufacture". Forging \& Stamping Technology, vol.34, no. 5, pp. 55-57, 2009

[10] Zhao Yun Hao, Li Yan Li. "Technology and application of spinning ". Bei Jing: Machinery Industry Press, pp. 51-52, 2007 